\title{
D-STATCOM predictive direct power control research under unbalanced voltage of power system
}

\author{
TianYi QU \\ School of Management \\ Xu-Zhou Institute of Technology \\ Xu-Zhou, Jiangsu, China 221008 \\ jdbh2001@163.com
}

\author{
Hao PENG \\ School of Information and Electrical Engineering \\ CUMT \\ Xu-Zhou, Jiangsu, China 221008 \\ ph5798819@qq.com
}

\begin{abstract}
Predictive direct power control strategy is led into the research of distribution network D-STATCOM. When the grid voltage is asymmetrical, the 2 times frequency instantaneous power pulsation of PPC will cause D-STATCOM output current total harmonic distortion rate higher. After analyzing the relationship of voltage and harmonic content rate, a method is posed. In the method, the instantaneous power feedback value can be calculated through the sample positive sequence components extracted by the instantaneous symmetrical components. Then a power predictive model can be deduced according to the positive sequence equivalent circuit. The positive sequence power substituted the full power control to achieve the control of compensation current amplitude and phase. The method can eliminate the device overflow threat and ensure output current three-phase symmetry. According to the simulation and experimental results, the method is feasible and practical.
\end{abstract}

Keywords-predictive direct power control; instantaneous power pulsation; harmonic total distortion rate; instantaneous symmetry components

\section{INTRODUCTION}

People' s requirements for the reliability and quality of the power supply of distribution system become higher. Distribution Static Synchronous Compensator (D-STATCOM), an important member of Flexible AC Transmission System (FACTS), has become a point in recent years. For it can effectively dissolve the power quality problems of voltage fluctuation and flicker and the low load power factor. The traditional D-STATCOM Controller is usually based on the hypothesis that three-phase voltage is symmetrical. While the phenomenon of asymmetric voltage in distribution system commonly exists, so the traditional control strategy using rotating coordinate transformation and reactive current staggered decoupling control must be improved. Direct power control is widely used to control three phase grid-connected inverter and PWM rectifier, for it has excellent static and dynamic performance and the decoupling method of active and reactive power in stationary coordinate system.

When direct power control is used in three-level D-STATCOM reactive compensation control of the unbalanced grid, the instantaneous power pulsation of PCC will cause the total harmonic distortion of device output current higher, especially the third and fifth harmonic are abundant. There are lots of harm to the grid. It could increase power consumption, shorten the life of equipment, make the ground protection and remote control function abnormal, overheat the power grid and so on. Reference [4] used P-DPC algorithm to map grid negative sequence to inhibit D-STATCOM device overflow, but it neglects the influence that the instantaneous power at the point of common coupling pulsation exert on the device output current. The method of Reference [5] can eliminate the negative sequence current, active fluctuations and the three reactive power by adding different power compensation. While the negative sequence voltage component value is small, and the extraction accuracy errors still exist, so the error may be enlarged when calculating power compensation items. Reference [1] use VPI (vector proportional integrator) controller to adjust a specific frequency signal, and use close-loop control to eliminate the negative sequence and harmonic components. But when it is used for D-STATCOM, the design appears complex.

This paper deduced a prediction model of positive sequence power, mapping model algorithm to the inverter AC side to suppress overcurrent device. P-DPC reduced the output current harmonic distortion and suppressed the adverse effects of negative sequence voltage.

\section{THREE-LEVEL D-STATCOM VOLTAGE ASYMMETRY OPERATION ANALYSIS}

Fig. 1 shows the schematic diagram that P-DPC control D-STATCOM to compensate load reactive power. According to the control period voltage $e_{\mathrm{s}}$, inverter output current ${ }^{i}$, DC side capacitor voltage $V_{\mathrm{dc} 1}$ and the samples of $V_{\mathrm{dc} 2}$, the feedback value of particular active power and reactive power and DC side active power given value can be calculated. The power deviation can be known, then the output voltage of inverter in next circle could be calculated.

There is not enough space to derive the specific control algorithm. The control equation is shown in (1). 


$$
\begin{aligned}
& {\left[\begin{array}{l}
u_{\alpha}(k) \\
u_{\beta}(k)
\end{array}\right]=\frac{L}{T_{s}\left\|\dot{e}_{s}\right\|^{2}}\left[\begin{array}{cc}
e_{s \alpha}(k) & e_{s \beta}(k) \\
e_{s \beta}(k) & -e_{s \alpha}(k)
\end{array}\right] \times} \\
& {\left[\begin{array}{l}
P^{*}(k+1)-P(k) \\
Q^{*}(k+1)-Q(k)
\end{array}\right]+\left[\begin{array}{c}
e_{s \alpha}(k) \\
e_{s \beta}(k)
\end{array}\right]}
\end{aligned}
$$

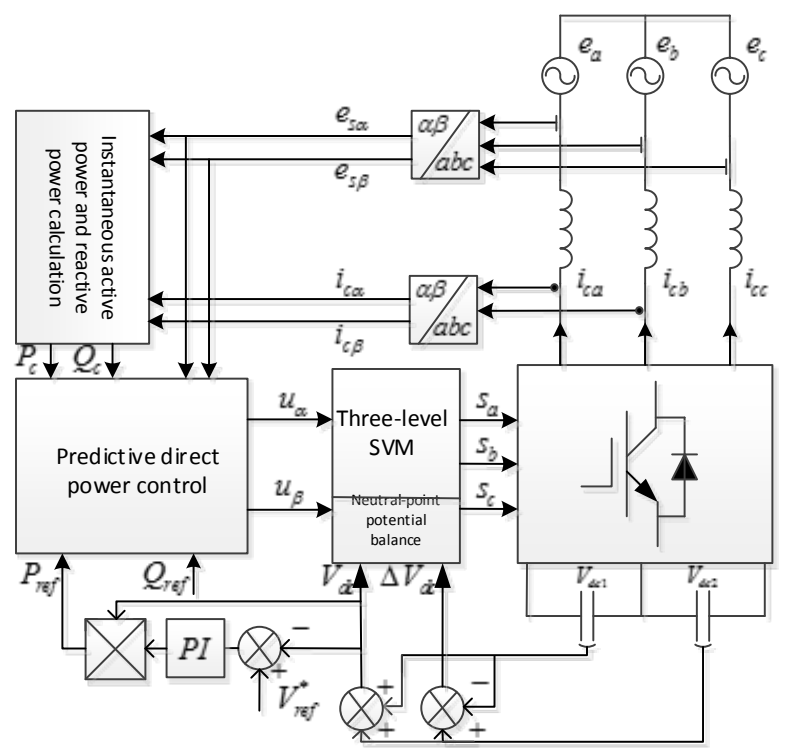

Fig. 1 Three-level D-STATCOM schematic diagram

There are two hypothesis : 1 ) the voltage unbalance factor does not exceed the maximum unbalance factor $K_{m}$ which is mentioned in Reference [4], which means the modulation has not appeared in the system; 2) use the method mentioned in Reference [8] to suppress the influence that DC side voltage fluctuation exert on AC side. The grid voltage of common connection and D-STATCOM output current can be expressed with the equation in the static $\alpha \beta$ coordinate.

$$
\begin{aligned}
& e_{s \alpha \beta}=E_{P} e^{j(\omega t+\gamma)}+E_{N} e^{-j(\omega t+\delta)} \\
& i_{c \alpha \beta}=I_{P} e^{j(\omega t+\phi)}
\end{aligned}
$$

According to (2), the instantaneous complex power formula is shown in (3).

$$
\begin{aligned}
& s=p+j q=e_{s \alpha \beta} i^{*} \alpha \beta \\
& =E_{P} I_{P} e^{j(\gamma-\phi)}+E_{N} I_{P} e^{-j(2 \omega t+\delta+\phi)}
\end{aligned}
$$

In (3) $E_{P}, E_{N}$ are respectively the amplitude of positive sequence and negative sequence parameter of three-phase grid voltage. $I_{P}$ is the amplitude of D-STATCOM output current positive parameter.

Expand it to get (4).

$$
\begin{aligned}
& p=\bar{p}+\hat{p}=e_{P} i_{P} \cos (\gamma-\phi)+e_{N} i_{P} \cos (2 \omega t+\delta+\phi) \\
& q=\bar{q}+\hat{q}=e_{P} i_{P} \sin (\gamma-\phi)-e_{N} i_{P} \sin (2 \omega t+\delta+\phi)
\end{aligned}
$$

$\bar{p} 、 \hat{p}$ and $\bar{q}, \hat{q}$ are DC component and fluctuation component of instantaneous active power and reactive power. Then active power and reactive power fluctuation rate are shown in (5).

$$
\begin{aligned}
& \Delta p=\left|\frac{p-\bar{p}}{\bar{p}}\right|_{\max }=\frac{\varepsilon}{\cos (\gamma-\phi)} \\
& \Delta q=\left|\frac{q-\bar{q}}{\bar{q}}\right|_{\max }=\frac{\varepsilon}{\sin (\gamma-\phi)}
\end{aligned}
$$

$\varepsilon=e_{P} / e_{N}$ is the grid voltage unbalanced degree. The active power and reactive power fluctuation rate are proportional to the grid voltage unbalanced degree. Bring (5) in (4) to get (6).

$$
\begin{aligned}
& p=\bar{p}+\Delta p \bar{p} \cos (2 \omega \mathrm{t}+\delta+\phi) \\
& q=\bar{q}-\Delta q \bar{q} \sin (2 \omega \mathrm{t}+\delta+\phi)
\end{aligned}
$$

The system control-period is $T_{s}$, after discreting bring it in (1) to get (7).

$$
\begin{aligned}
& {\left[\begin{array}{l}
u_{\alpha}(k) \\
u_{\beta}(k)
\end{array}\right]+\left[\begin{array}{l}
\Delta u_{\alpha}(k) \\
\Delta u_{\beta}(k)
\end{array}\right]=\left[\begin{array}{l}
e_{s \alpha}(k) \\
e_{s \beta}(k)
\end{array}\right]+\frac{L}{T_{s}\left\|\dot{e}_{s}\right\|^{2}} \times} \\
& {\left[\begin{array}{cc}
e_{s \alpha}(k) & e_{s \beta}(k) \\
e_{s \beta}(k) & -e_{s \alpha}(k)
\end{array}\right] \times\left[\begin{array}{c}
P^{*}(k+1)-\bar{p}(k) \\
Q^{*}(k+1)-\bar{q}(k)
\end{array}\right]}
\end{aligned}
$$

In (7),

$$
\begin{aligned}
& {\left[\begin{array}{c}
\Delta u_{\alpha}(k) \\
\Delta u_{\beta}(k)
\end{array}\right]=\frac{L}{T_{s}\left\|\dot{e_{s}}\right\|^{2}}\left[\begin{array}{cc}
e_{s \alpha}(k) & e_{s \beta}(k) \\
e_{s \beta}(k) & -e_{s \alpha}(k)
\end{array}\right]} \\
& {\left[\begin{array}{c}
\Delta p \bar{p}(k) \cos \left(2 \omega k T_{s}+\delta+\phi\right) \\
-\Delta q \bar{q}(k) \sin \left(2 \omega k T_{s}+\delta+\phi\right)
\end{array}\right]}
\end{aligned}
$$
(8).

Bring voltage space vector to the upper equation to get

$$
\begin{aligned}
\Delta u_{\alpha \beta}= & f\left[( \overline { p } ( k ) \Delta p + \overline { q } ( k ) \Delta q ) \left(e_{P} e^{j(30 t+\gamma+\delta+\phi)}\right.\right. \\
& \left.+e_{N} e^{j(\omega t+\phi)}\right)+(\bar{p}(k) \Delta p-\bar{q}(k) \Delta q) \\
& \left.\left(e_{P} e^{-j(\omega t++\delta+\phi-\gamma)}+e_{N} e^{-j(30 t+2 \delta+\phi)}\right)\right]
\end{aligned}
$$

$$
f=\frac{L}{2 T_{s}\left\|e_{s}\right\|^{2}}
$$


Equation (8) shows that the instantaneous power two-times frequency fluctuation of PPC will map positive and negative sequence harmonic component and negative sequence based component on the inverter AC side when the grid voltage is unbalanced. For reactive fluctuation component amplitude is little compared with DC component, and the ideal active and reactive power fluctuation amplitude are approximately equal, (9) can be deduced.

$$
\begin{array}{r}
\Delta u_{\alpha \beta}=f(\bar{p}(k) \Delta p+\bar{q}(k) \Delta q) \\
\left(e_{P} e^{j(3 \omega t+\gamma+\delta+\phi)}+e_{N} e^{j(\omega t+\phi)}\right)
\end{array}
$$

Third harmonic voltage make D-STATCOM output current contain third harmonic current, then causing PPC instantaneous power four times pulsation. And more harmonic current will be introduced after P-DPC mapping, causing D-STATCOM device output current distorted.

\section{PREDICTIVE DIRECT POWER CONTROL STRATEGY}

According to (3), when the grid voltage is definite, the instantaneous power DC component of D-STATCOM compensation current is enough to describe the reactive compensation current's amplitude and phase information. So controlling instantaneous power DC component can control the compensation current which comes from the control device. Based on this, the predictive direct power control strategy is proposed, using instantaneous symmetrical component method to decompose voltage and current samples. Extract the positive sequence component for calculating the instantaneous power feedback value. The positive sequence power predictive model can be derived according to D-STATCOM positive sequence net circuit. The grid negative sequence voltage can be mapped to the inverter AC side to suppress the current by using power prediction model algorithm, to ensure the output offset current three-phase balanced and undistorted. The positive and negative sequence network is shown in Fig.2.

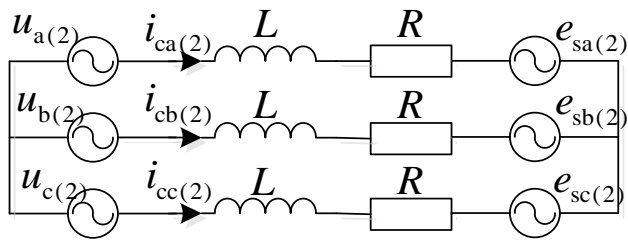

(a) Positive sequence network equivalent circuit

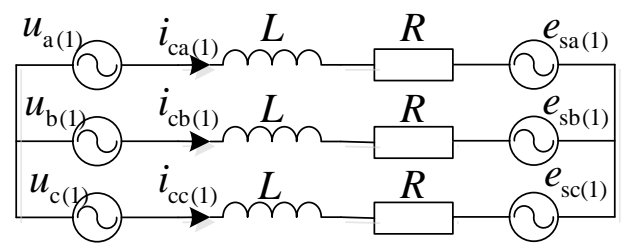

(b) Negative Positive sequence network equivalent circuit

Fig. 2 Predictive direct power control D-STATCOM sequence network

If the system sample period is $T_{\mathrm{s}}$, considering $T_{\mathrm{s}}$ is much smaller than the grid voltage period, the positive voltage in adjacent periods can be considered the same. The positive sequence complex power's change in the $\mathrm{k}$ sample period can be calculated in (10).

$$
\left[\begin{array}{c}
\Delta p(k) \\
\Delta q(k)
\end{array}\right]=\left[\begin{array}{cc}
e_{s \alpha(1)}(k) & e_{s \beta(1)}(k) \\
e_{s \beta(1)}(k) & -e_{s \alpha(1)}(k)
\end{array}\right]\left[\begin{array}{c}
i_{c \alpha(1)}(k+1)-i_{c \alpha(1)}(k) \\
i_{c \beta(1)}(k+1)-i_{c \beta(1)}(k)
\end{array}\right]
$$

While this paper's theme is low voltage distribution network, the AC side resistance can be ignored. The state formula of positive sequence network equivalent circuit can be forward difference discrete.

$$
\left[\begin{array}{l}
i_{\mathrm{c} \alpha}(k+1)-i_{\mathrm{c} \alpha}(k) \\
i_{\mathrm{c} \beta}(k+1)-i_{\mathrm{c} \beta}(k)
\end{array}\right]=\frac{T_{\mathrm{s}}}{L}\left[\begin{array}{l}
u_{\alpha}(k)-e_{\mathrm{s} \alpha}(k) \\
u_{\beta}(k)-e_{\mathrm{s} \beta}(k)
\end{array}\right]
$$

Bring (11) into (10), then add the grid negative voltage to both side of equation.

$$
\begin{aligned}
& {\left[\begin{array}{l}
u_{\alpha(1)}(k) \\
u_{\beta(1)}(k)
\end{array}\right]+\left[\begin{array}{l}
e_{s \alpha(2)}(k) \\
e_{s \beta(2)}(k)
\end{array}\right]=\left.\frac{L}{T_{s} \| \cdot e_{s(1)}}\right|^{2}\left[\begin{array}{cc}
e_{s \alpha(1)}(k) & e_{s \beta(1)}(k) \\
e_{s \beta(1)}(k) & -e_{s \alpha(1)}(k)
\end{array}\right]} \\
& x\left[\begin{array}{l}
P^{*}(k+1)-P(k) \\
Q^{*}(k+1)-Q(k)
\end{array}\right]+\left[\begin{array}{l}
e_{s \alpha(1)}(k) \\
e_{s \beta(1)}(k)
\end{array}\right]+\left[\begin{array}{l}
e_{s \alpha(2)}(k) \\
e_{s \beta(2)}(k)
\end{array}\right]
\end{aligned}
$$

Then combine with the equation $\left[\begin{array}{l}u_{\alpha(2)}(k) \\ u_{\beta(2)}(k)\end{array}\right]=\left[\begin{array}{l}e_{s \alpha(2)}(k) \\ e_{s \beta(2)}(k)\end{array}\right]$

is (13).

$$
\begin{aligned}
{\left[\begin{array}{l}
u_{\alpha}(k) \\
u_{\beta}(k)
\end{array}\right]=\frac{L}{\left.T_{\mathrm{s}} \| \begin{array}{cc}
e_{e_{\mathrm{s} \alpha(1)}}(k) & e_{\mathrm{s} \beta(1)}(k) \\
e_{\mathrm{s} \beta(1)}(k) & -e_{\mathrm{s} \alpha(1)}(k)
\end{array}\right]} } \\
\times\left[\begin{array}{l}
P^{*}(k+1)-P(k) \\
Q^{*}(k+1)-Q(k)
\end{array}\right]+\left[\begin{array}{l}
e_{\mathrm{s} \alpha}(k) \\
e_{\mathrm{s} \beta}(k)
\end{array}\right]
\end{aligned}
$$

In (13) the positive reference is used to supply DC side voltage, the negative reference is to compensate load negative power. And the grid negative sequence voltage which is mapped to inverter AC side suppresses device overcurrent problem by the equation. The voltage modulation signal can be considered as three-level space vector PWM input, the PWM driving signal controls the IGBT module to turn on or off.

\section{EXPERIMENT}

To examine the method, a system experiment platform was built, the parameters were in Table 1 . 
TABLE 1 EXPERIMENT MODULE PARAMETERS

\begin{tabular}{cc}
\hline \hline parameters & Value \\
\hline $\begin{array}{c}\text { Three-phase system line voltage } \\
\text { effective value/V }\end{array}$ & 190 \\
DC side capacitance voltage /V & \\
DC side capacitance/mF & 300 \\
AC side equivalent coupling & 4.8 \\
inductance/mH & 6 \\
AC side equivalent coupling & \\
$\quad$ resistance / $\Omega$ & 0.02 \\
$\begin{array}{c}\text { Switch frequency/kHz } \\
\text { Sample frequency/kHz }\end{array}$ & 5 \\
DSTATCOM reactive power \\
compensation range/kvar
\end{tabular}

The normal voltage unbalanced degree allowable value of power system public junction is $2 \%$, short time is not permitted to exceed $4 \%$. To examine the harmonic current suppressing effect, we chose D-STATCOM device output current third harmonic's content rate and THD which were from different grid voltage unbalanced degree. Then the result was compared with the simulation result of Reference [4]. The simulation results are shown in Table 2.

TABLE 2 SIMULATION RESULTS

\begin{tabular}{c|c|c|c|c}
\hline \hline $\begin{array}{c}\text { Voltage } \\
\text { unbalance } \\
\text { degree }\end{array}$ & \multicolumn{2}{|c|}{$\begin{array}{c}\text { Traditional predictive direct } \\
\text { power control }\end{array}$} & $\begin{array}{c}\text { Positive sequence predictive } \\
\text { model P-DPC }\end{array}$ \\
\cline { 2 - 5 } $\boldsymbol{\varepsilon} / \%$ & $\begin{array}{c}\text { Third } \\
\text { harmonic } \\
\text { content rate } \\
/ \%\end{array}$ & $\begin{array}{c}\text { Total } \\
\text { harmonic } \\
\text { distortion } \\
\text { rate/\% }\end{array}$ & $\begin{array}{c}\text { Third } \\
\text { harmonic } \\
\text { content rate } \\
/ \%\end{array}$ & $\begin{array}{c}\text { Total } \\
\text { harmonic } \\
\text { distortion } \\
\text { rate/\% }\end{array}$ \\
\hline 0.5 & 0.63 & 1.84 & 0.03 & 1.13 \\
1 & 1.16 & 2.12 & 0.03 & 1.10 \\
1.5 & 1.69 & 2.47 & 0.09 & 1.09 \\
2 & 2.27 & 2.88 & 0.03 & 1.2 \\
3 & 3.38 & 3.80 & 0.09 & 1.18 \\
4 & 4.52 & 4.85 & 0.23 & 1.14 \\
\hline
\end{tabular}

Fig. 3 is the D-STATCOM output current spectrogram of two kinds of control strategy. Fig.4 is the power response curve which is after D-STATCOM compensation controlled by the positive sequence power prediction model.
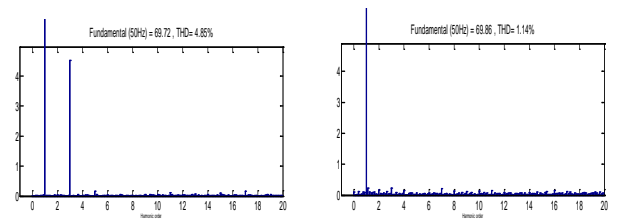

Fig. 3 D-STATCOM output current spectrogram

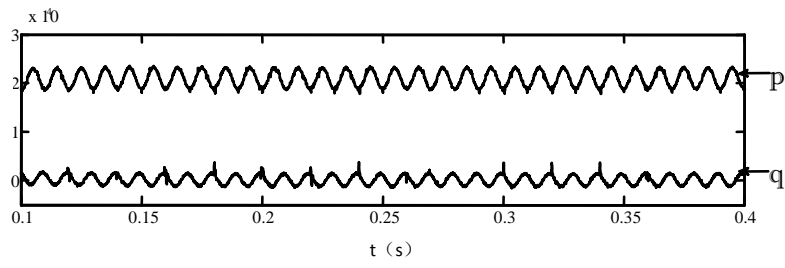

Fig. 4 Instantaneous power response curve

\section{CONCLUSIONS}

(1) Direct power control strategy based on positive sequence power predictive module just need extract the positive sequence component, so it can select the quicker sequence decomposition method when the precision condition is met.

(2) Controlling the PPC positive sequence power component can control output reactive compensation-current, and simultaneously suppress the output current harmonic distortion of instantaneous power.

(3) The grid negative sequence voltage mapped to the inverter AC side by the power predictive model can eliminate the device overflow threat and ensure output current three-phase symmetry.

\section{REFERENCES}

[1] Heng Nian, Yongbo SHEN, Yipeng SONG, "Direct power control strategy of grid connected inverter under unbalanced and harmonic grid voltage,” Power System Technology,2014,38(6):1452-1458

[2] Aguilera R P, Quevedo D E, Vazquez S , Franquelo L."Generalized predictive direct power control for ac/dc converters," ECCE Asia Downunder (ECCE Asia), 2013 IEEE,Melbourne, VIC,2013.

[3] Aurtenechea S, Rodriguez M, Oyarbide E , Torrealday J."Predictive direct power control of mv grid-connected three-level npc converters", IEEE International Symposium on Industrial Electronics, ISIE, Vigo, 2007.

[4] Yang ZHOU, Daozhuo JIANG, Yufen WANG, "Study on direct power control of static synchronous compensator based on virtual flux linkage,” Power System Technology ,2012,36(11):205-210.

[5] Xugang GUO, Zengwei GAO, Jingyuan YIN, Xinmin JIN, Tianyi MA, "Predictive Direct Power Control for PWM Rectifier Under Unbalanced Grid Voltage Conditions," Power System Technology 2013,37(8):2362-2367.

[6] Xufeng YUAN, Shijie CHENG, Jinyu WEN, “An improved method of instantaneous symmetrical components and its detection for positive and negative sequence current,” Proceedings of the CSEE ,2008,28(1):52-58

[7] Bouafia A, Gaubert J-P , Krim F. "Predictive direct power control of three-phase pulsewidth modulation (PWM) rectifier using space-vector modulation (SVM)," IEEE Transactions on Power Electronics 2010,25(1):228-236.

[8] Chunming TU, Hui LI, Jie TANG, An LUO, “Analysis and suppression of influence on d-statcom under unbalanced voltage of power system," Transactions of China Electrotechnical Society,2009,24(10):114-121 